\title{
P01-200
}

\section{ALL AVAILABLE TREATMENTS OF BIPOLAR DISORDERS}

P. Misic, D. Arandjelovic, S. Stanojkovic, S. Vladejic, J. Mladenovic

Forensic Psychiatric Centre, Specialized Hospital For Psychiatric Disorders 'Gornja Toponica', Nis, Serbia

Bipolar disorder is a treatable brain disorder that causes changes in person's mood, thought, energy and behaviour.

All available resources were searched and combined with the experince from everyday practice in Specialized Psychiatric Hospital "Gornja Toponica" and this is a rewiev of the various treatments that are currently available for the treatment of bipolar disorders.

Because bipolar disorder is a recurrent illness, long-term preventive treatment is strongly recommended and almost always indicated. A strategy that combines medication and psychosocial treatment is optimal for managing the disorder over time. In situations where medication, psychosocial treatment, and the combination of these interventions prove ineffective, or work too slowly to relieve severe symptoms such as psychosis or suicidality, Electroconvulsive therapy (ECT), Transcranial Magnetic Stimulation (TMS), Vagus Nerve Stimulation (VNS), Magnetic Stimulation Therapy (MST) and even herbal or natural supplements may be considered.

Lithium, the first mood-stabilizing medication is often very effective in controlling mania and preventing the recurrence of both manic and depressive episodes.

Anticonvulsant medications, such as valproate or carbamazepine, also can have mood-stabilizing effects.

Newer anticonvulsant medications, including lamotrigine, gabapentin, and topiramate, are also very effective in stabilizing mood cycles.

Atypical antipsychotic medications, including clozapine, olanzapine, risperidone, quetiapine, and ziprasidone, are are also used for bipolar disorder treatment.

If insomnia is a problem, a high-potency benzodiazepine medication such as clonazepam or lorazepam may be helpful to promote better sleep. However, since these medications may be habit-forming, they are best prescribed on a short-term basis. Other types of sedative medications, such as zolpidem, are sometimes used instead. 